\title{
M-EPAR to Improve the Quality of the MANETs
}

\author{
Er.Harjinder singh, Er. Preet kanwal kaur \\ Department of ECE Student, Ludhiana College of Engineering and Technology India \\ Department of ECE Associate Professor, Ludhiana College of Engineering and Technology,India
}

\begin{abstract}
In MANET, power aware is important challenge issue to improve the communication energy efficiency at individual nodes. We propose modified efficient power aware routing (M-EPAR), a new power aware routing protocol that increases the network lifetime of MANET. Designing a power aware routing algorithm or technique is one of the most important point considered in Mobile Ad Hoc Networks. These nodes are driven by reactive protocols where broadcasting is mandatory to form a path between two nodes. So in case of death of the node resulting out of less battery calls for re-routing. Since many existing techniques focuses on energy aware routing this paper presents combination of energy aware routing merged with link quality determined by packet drop rate. The proposed scheme has outperformed the existing technique in terms of packet delivery ratio, throughput and energy consumption.
\end{abstract}

Keywords: Power aware routing, packet drop, MANETs, throughput.

\section{INTRODUCTION}

Wireless network is divided in to two types of networks infrastructure and infrastructure less networks. In infrastructure networks communications among nodes are established and maintained through centralized controllers. Infrastructure less network is commonly referred to as wireless ad hoc network. This type of a network is organized in an ad hoc manner, where nodes are capable of making connections between them and communicate with each other in a multi-hop manner. MANET is group of mobile nodes that form a network by connecting each other with wireless links, independent of any centralized administration. Each mobile nodes act as a host and router and forward packets to other nodes. Due to the moving nature of mobile nodes in this network continuously changes its network topology Mobile ad hoc networks (MANETs) are self-sorting out, independent systems that do not need any centralized controller. Two nodes communicate directly if they're within the transmission range, As a result of their distinctive edges and versatilities, MANETs have a good variety of applications like cooperative, distributed mobile computing (e.g., sensors, conferences), disaster relief (e.g., flood, earthquake), war front activities and communication between vehicles on highways. Most of those applications demand multicast or cluster communication [6]. The routing in Mobile Ad Hoc Networks is usually guided by routing protocols that do not take into account residual energy of the nodes. In other terms the traditional routing protocols are not energy efficient. In case of formation of the path with low energy nodes, the link breaks easily and rerouting is required. Thus preserving the energy of the nodes is most imperative issue for such networks.
This paper presents the brief survey of the existing techniques related to energy efficiency in section II. Then Section III presents the research gap which is modified by the method described in Section IV. Finally, the section V presents the results with the conclusion shown in the last section of the paper.

\section{LITERATURE SURVEY}

Researcher in [1] proposed energy efficient power aware routing (EPAR), a new power aware routing protocol that increases the network lifetime of MANET. In contrast to conventional power aware algorithms, EPAR identifies the capacity of a node not just by its residual battery power, but also by the expected energy spent in reliably forwarding data packets over a specific link. Using a mini-max formulation, EPAR selects the path that has the largest packet capacity at the smallest residual packet transmission capacity. This protocol must be able to handle high mobility of the nodes that often cause changes in the network topology. This paper evaluates three ad hoc network routing protocols (EPAR, MTPR, and DSR) in different network scales, taking into consideration the power consumption. Indeed, our proposed scheme reduces for more than $20 \%$ the total energy consumption and decreases the mean delay, especially for high load networks, while achieving a good packet delivery ratio.

This paper [2] attempts to modify the popular on demand routing protocol AODV to make it energy aware. The proposed algorithm also varies the transmission power between two nodes as per their distance. The protocols are simulated using Network Simulator (NS-2.34). The performance of both the protocols is analysed under various 
conditions and the proposed scheme shows efficient energy utilization and increased network lifetime.

In this paper [3], a cross-layer optimized energy-aware multipath routing protocol (EMRP) for mobile ad hoc networks (MANET) is proposed. By sharing the information among the physical layer, the MAC sub-layer and the network layer, EMRP efficiently utilizes the network resources such as the node energy and the link bandwidth. Simulation results show that the protocol prolongs the network lifetime, increases the volume of packets delivered, lowers the energy dissipation per bit of data delivery and shortens the end-to-end delay.

This research [4] proposed Maximum Minimum (MAX-MIN) Energy based Routing scheme selecting the nodes in routing that is having the sufficient energy for routing packets and data packets in network. The MAX-MIN scheme routing strategies are scalable because the protocol can minimize the energy consumption under not just some specific operative conditions such as lower mobility, light traffic load or low number of node but also in dense and loaded network. This means that the design of an energy efficient routing protocol should consider also scalability issue in order to apply it in wider scenarios and to be sure that the MAX-MIN performance do not degrade too much when the energy degrades because of set of energy threshold value. In proposed scheme routing protocol either to route data through the path with maximum energy or to minimize the routing overhead, end to end delay and improves network life time of network.

This paper [5] employs the Binary Particle Swarm Optimization algorithm (BPSO) to add the energy awareness feature to the TORA routing protocol. The proposed protocol considers routes length in its route selection process and also includes routes energy level in its calculations. It formulates the routing issue as an optimization problem and then employs BPSO to choose a route that maximizes a weighted function of the route length and the route energy level. Extensive simulations in ns-2 simulator environment show that the proposed routing protocol, called BPSO-TORA, prolongs the network lifetime remarkably and outperforms TORA in terms of network life time, system life time and total delivered data.

\section{RESEARCH GAP}

In the work done by the authors in [1], the authors have designed a new algorithm. According to it, the authors tend to choose low power congested paths by employing the min-max algorithm. They tend to choose that path to send data from source to destination in which the minimum energy of the nodes is highest among other possible paths. This would make certain that the network's performance is fair in terms of energy consumed. However, the topology changes must be factored in the path selection procedure so that the data communication between source and destination is smooth and less packets gets dropped.

\section{PROPOSED WORK}

The proposed work will aim at reducing the energy consumed during the route finding process by taking into account two parameters, namely remaining energy of the nodes as well as their drop rate. If any node is dropping more number of packets during the route request phase, that would mean the node is not fit to forward the data packets during the data transmission from source to destination node. Also, since the energy consumption is the prime focus, so the remaining energy of the nodes is also considered as the factor to decide the nodes eligible for forwarding the route request packets. So, when the source node starts the process of finding the path to the destination node, only those nodes would forward the route request for which the objective function is less than the objective function of the same hop nodes. Here, the term same hop nodes refer to all those nodes whose hop count is same with respect to the source node. When the route request reaches the destination node, the path will be chosen according to the min-max algorithm described in the existing work.

$\mathrm{f}(\mathrm{e}, \mathrm{d})=\mathrm{E}(\mathrm{i}) * \mathrm{We}+\mathrm{D}(\mathrm{i}) * \mathrm{Wd}$

Where E represents energy consumed by the node And D represents its drop rate

\section{RESULTS}

The proposed scheme as well as the existing scheme, EPAR, were simulated in open source software, NS2.35. The simulation parameters used in the work are defined below:

\begin{tabular}{|c|c|}
\hline Parameter & Value \\
\hline Channel & Wireless \\
\hline Propagation Model & Two Ray Ground \\
\hline Mac & 802.11 \\
\hline Queue & CMU \\
\hline Antenna & Omni Directional \\
\hline Mobility Model & Random Way Point \\
\hline Routing Protocol & DSR,EPAR,MEPAR \\
\hline
\end{tabular}




\begin{tabular}{|c|c|}
\hline Number of nodes & 120 \\
\hline Initial Energy & 7.1 Joules \\
\hline Network Area & $2000 \mathrm{~m} * 2000 \mathrm{~m}$ \\
\hline
\end{tabular}

Table I: Simulation Parameters

Fig.1 shows the comparison of the packet delivery ratio for both the schemes. The proposed scheme has found to give better values for this parameter. The values for the proposed scheme is found to be hovering near 65 percent and the for the existing scheme it was found to be near 55 percent. Fig. 2 shows the comparison of the average energy remaining in the network at the end of the simulation time. Initially 50 nodes were supplied with energy of 7.1 Joules and remaining nodes were supplied with 3.1 Joules, thus averaging to 5.1 Joules at the starting of the simulation time. At the end of the simulation the remaining energy for the proposed scheme was 5 Joules approximately and 3 Joules for the existing scheme. Thus it clearly indicates better network lifetime.

Fig.3 shows the comparison of the throughput values for both the schemes. Since the packet delivery ratio values showed higher performance, thus on the same lines, the throughput parameter also showed higher values for the proposed scheme. The value for the proposed scheme was approx. $40 \mathrm{Kbps}$, and for the existing scheme was 22 kbps

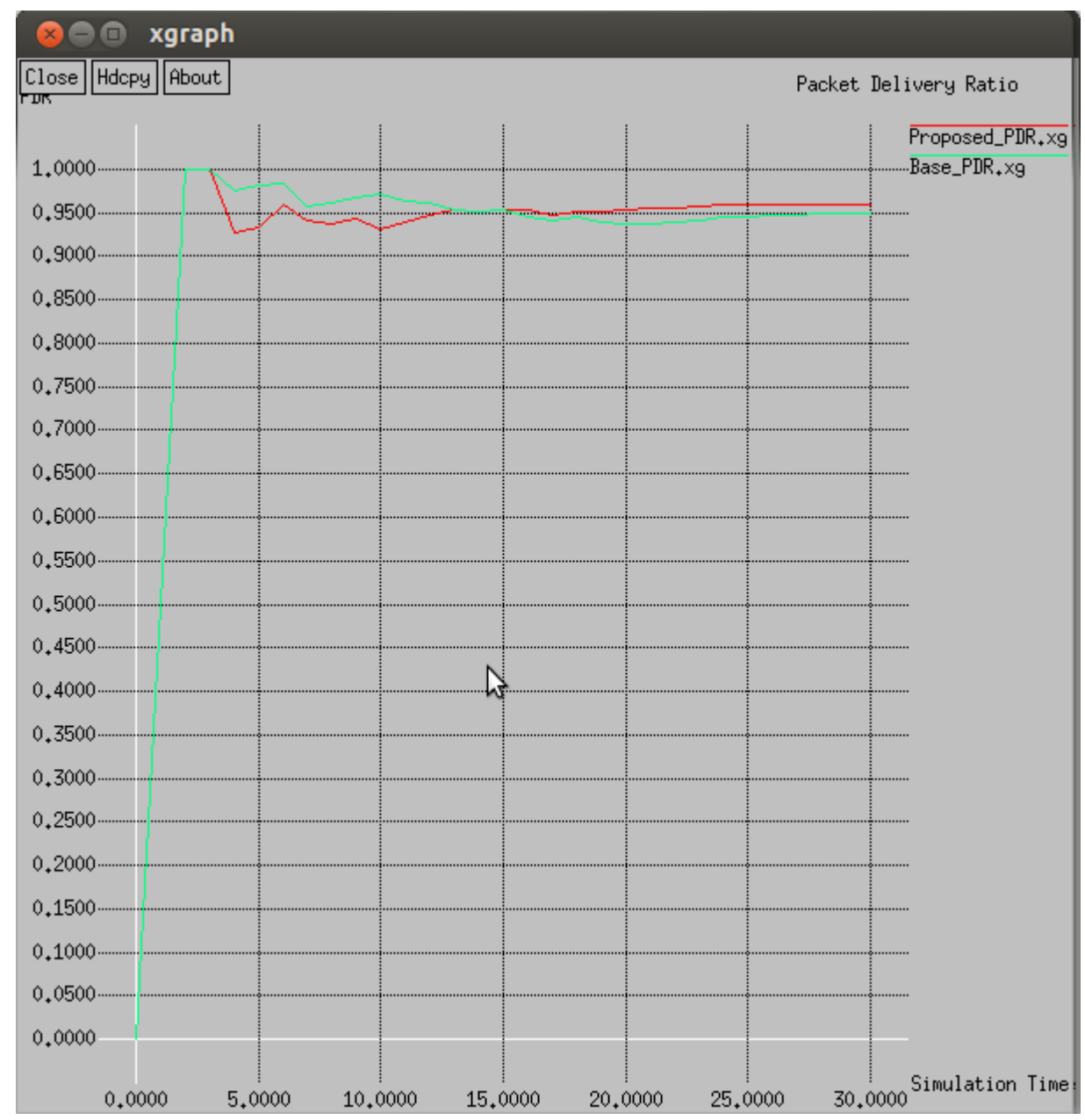

Fig.1: PDR Comparison 


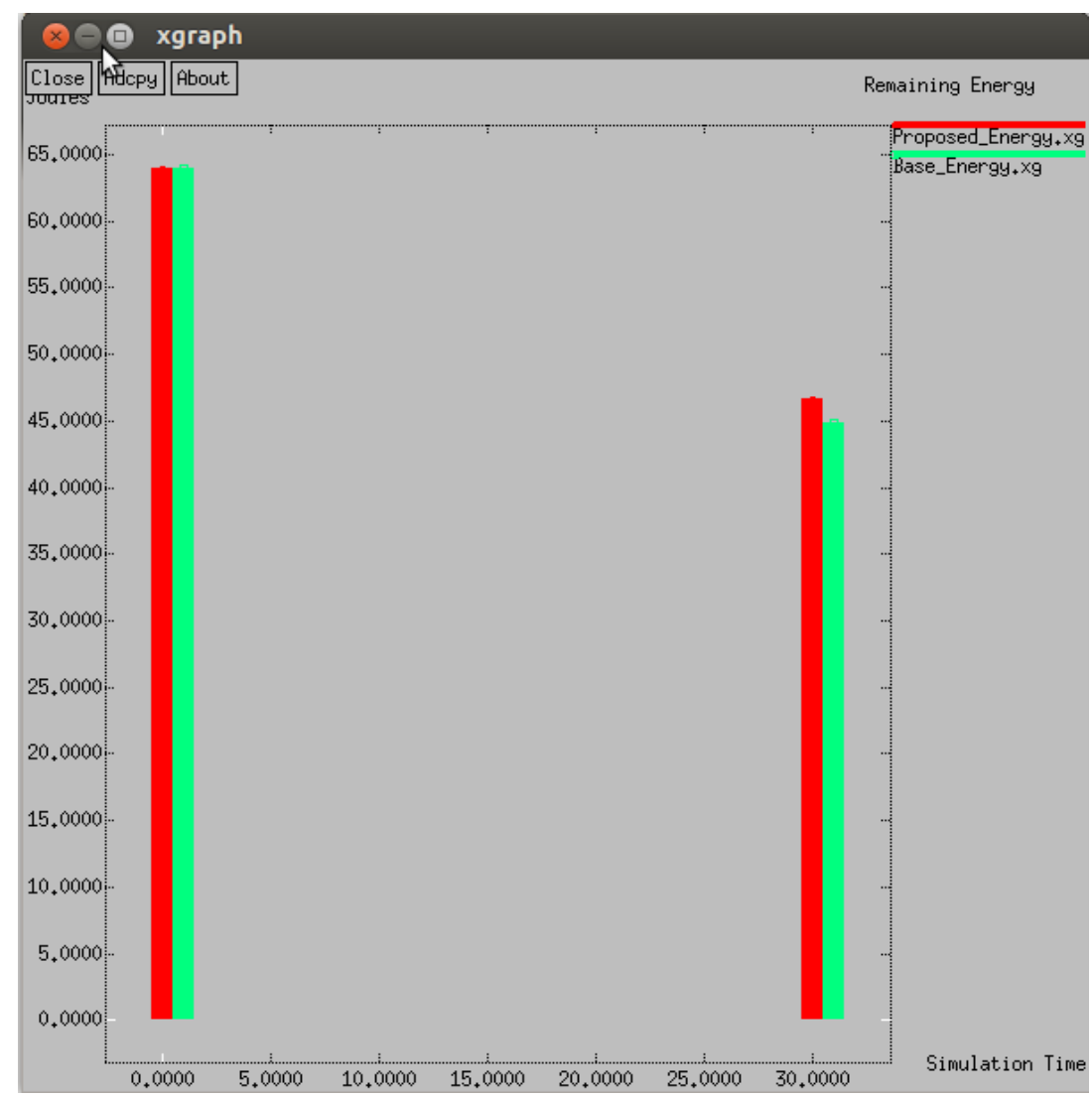

Fig.2 : Remaining energy Comparison

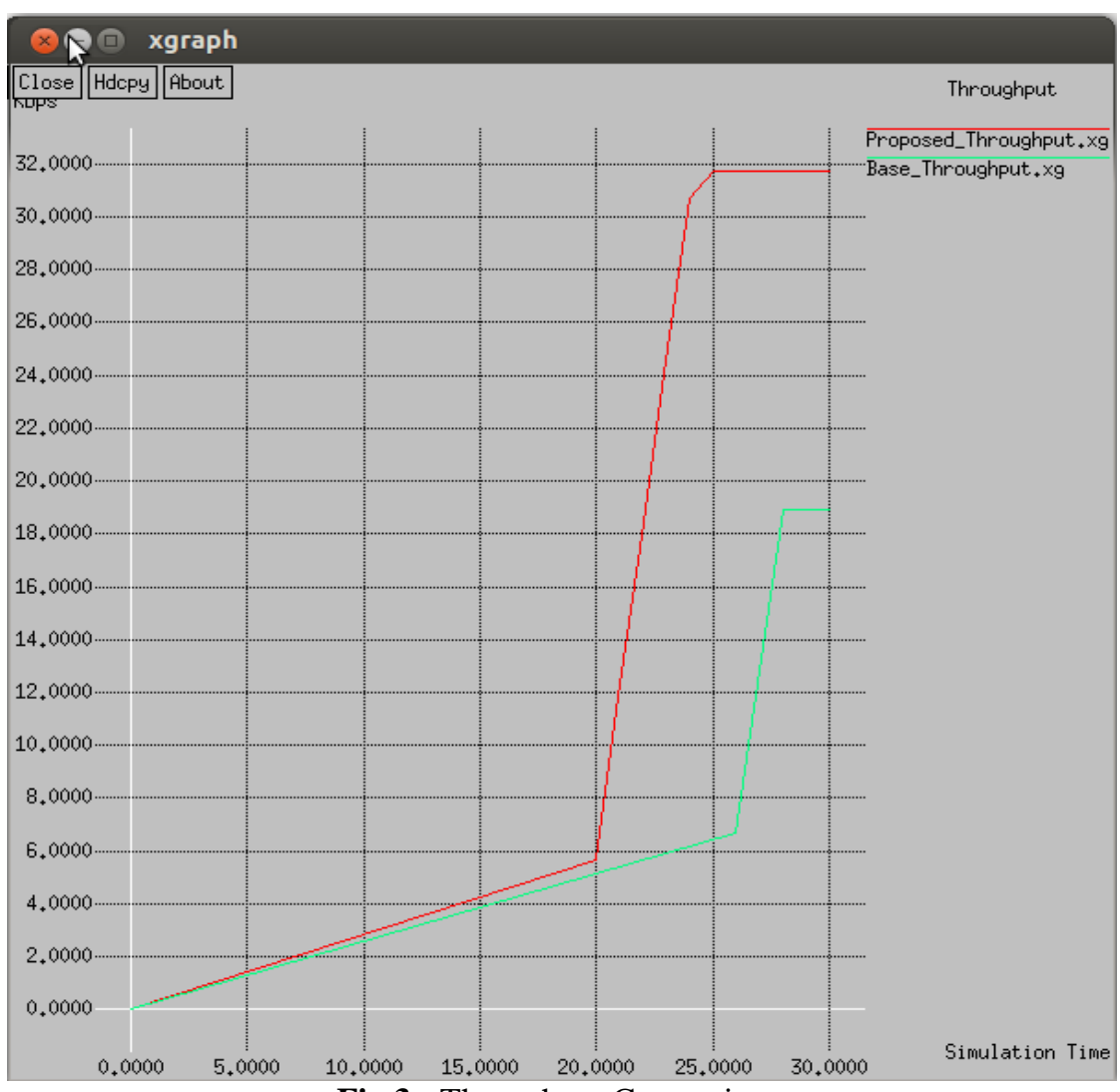

Fig.3 : Throughput Comparison 


\section{CONCLUSION AND FUTURE WORK}

The aim of the study was to improve the lifetime of the network by reducing the energy consumed by the nodes. The proposed scheme optimized the broadcasting process and the performance of the network was compared on the basis of remaining energy, throughput and packet delivery ratio. All the three parameter showed an improved performance over the existing scheme. While the value of packet delivery ratio was found to be approx. 65 percent, the same value for the existing scheme was nearly 55 percent. The credit to the better packet delivery ratio and throughput is given to the fact that during broadcasting only those nodes opt for the forwarding of Route Request messages who are fit or more superior to their neighboring nodes in terms of remaining energy and drop rate.

Since this study has taken into account fixed number of nodes in the network, the same work can be extended to analyze the performance of the network against varying number of nodes as well as varying mobility patterns.

\section{REFERENCES}

[1]. Shivashankar, HosahalliNarayanagowda Suresh, GollaVaraprasad and GuruswamyJayanthi, "Designing Energy Routing Protocol with Power Consumption Optimization in MANET", Volume 2, No. 2, June 2014 Emerging Topics in Computing, IEEE.

[2]. SeemaVerma, PinkiNayak and Rekha Agarwal, "Energy Efficient Routing in Mobile Adhoc Networks based on AODV Protocol", IJCSI International Journal of Computer Science Issues, Vol. 9, Issue 6, No 2, November 2012.

[3]. Meng Li, Lin Zhang, Victor O. K. Li, Xiuming Shan, Yong Ren, "An EnergyAware Multipath Routing Protocol for Mobile Ad Hoc Networks", ACM Sigcomm Asia'05, Apr. 10-12, 2005, Beijing, China.

[4]. Ashish Singh Baghel, VivekSuryawanshi, "MAX-MIN Energy based Routing Scheme for Reducing Power Consumption in MANET",International Journal of Advanced Research in Computer Science and Software Engineering, Volume 5, Issue 5, MAY 2015.

[5]. ShahramJamali, Leila Rezaei, SajjadJahanbakhshGudakahriz, "An Energy-efficient Routing Protocol for MANETs: A Particle SwarmOptimization Approach”,
Journal of Applied Research and Technology, Vol. 11, December 2013.

[6]. Nandkishor M. Pawar,Nandkishor P. Karlekar, "A Survey on Energy Efficient Routing Protocols in MANETS", International Journal of Computer Science and Mobile Computing, Vol.3 Issue.12, December2014, pg. 133-139. 